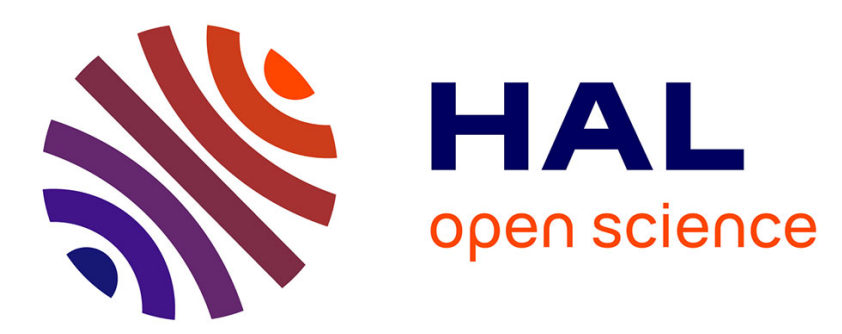

\title{
Automatic Atlas-Based Building of Point Distribution Model for Segmentation of Anatomical Structures from Brain MRI
}

Jonathan Bailleul, Su Ruan, Daniel Bloyet

\section{- To cite this version:}

Jonathan Bailleul, Su Ruan, Daniel Bloyet. Automatic Atlas-Based Building of Point Distribution Model for Segmentation of Anatomical Structures from Brain MRI. ISSPA'03 - IEEE Seventh International Symposium on Signal Processing and its Applications, 2003, Paris, France. pp.629 - 630, 10.1109/ISSPA.2003.1224960 . hal-00965352

\section{HAL Id: hal-00965352 \\ https://hal.science/hal-00965352}

Submitted on 30 Sep 2014

HAL is a multi-disciplinary open access archive for the deposit and dissemination of scientific research documents, whether they are published or not. The documents may come from teaching and research institutions in France or abroad, or from public or private research centers.
L'archive ouverte pluridisciplinaire HAL, est destinée au dépôt et à la diffusion de documents scientifiques de niveau recherche, publiés ou non, émanant des établissements d'enseignement et de recherche français ou étrangers, des laboratoires publics ou privés. 


\title{
AUTOMATIC ATLAS-BASED BUILDING OF POINT DISTRIBUTION MODEL FOR SEGMENTATION OF ANATOMICAL STRUCTURES FROM BRAIN MRI.
}

\author{
Jonathan Bailleul, Su Ruan, Daniel Bloyet \\ GREYC CNRS UMR 6072, Ensicaen \& Universite de Caen, F-14050 Caen cedex, France
}

\begin{abstract}
We propose a method based on a priori knowledge provided by anatomical atlases to build - almost - automatically a Point Distribution Model (PDM) of internal brain structures.

A 3D training set of shapes is constructed by registering chosen atlas over an MRI database, which is then landmarked using a method recently developed by Davies et al. This PDM global optimization process is driven by a Minimum Description Length (MDL) principle-based objective function.

Preliminary results of built PDMs are encouraging. Future work might build Active Appearance Models (AAM) from computed PDMs to set up a segmentation method.
\end{abstract}

\section{INTRODUCTION}

\subsection{Atlas-based MRI segmentation}

As a first attempt to segment anatomical structures from brain MRI volumes, we tried to improve the method [4] developed in our lab by J.H. Xue and relying on a priori information provided by an anatomical atlas Atlas $s_{a}$. Such an atlas results from segmentation of structures of interest ${ }^{1}$ in a reference MRI volume $\operatorname{Re} f_{a}$. This is a time-consuming and error-prone task which demands the intervention of anatomical experts, hence our limited number of such atlases (Cyceron, Harvard SPL, Talairach).

In that method, Atlas $s_{a}$ is first registered ${ }^{2}$ into a given patient MRI to infer fuzzy fields providing implicit spatial information about the expected positions of structures. Concurrently, patient MRI is oversegmented using Fuzzy Markov Random Fields into regions labeled among 20 fuzzy classes resulting from mixtures of anatomical brain tissues (CSF, GM, WM). Finally, a segmentation result is inferred sliceby-slice through conjunction of both sources using a rather stochastic GA approach ([4]) or, in our case, a sequence of amended morphologic filters - including hole-fill from a safe structure core - in an attempt to emphasize deterministic behavior and further extensibility.

\footnotetext{
${ }^{1}$ for now: ventricles, putamens, caudate nuclei, thalami, hippocampi

${ }^{2}$ Roger P. Woods's AIR, http://bishopw.loni.ucla.edu/AIR5/index.html
}

\subsection{Emerging need for a shape model}

In both cases, results show good global positioning and coverage rate relatively to expert-segmentation ${ }^{3}$. But we did not completely overcome local noticeable artifacts on lowcontrasted structure edges - ventricles excepted - that basically justified our new approach. This limitation incited us to look for a complementary approach emphasizing shapecorrectness of segmentation proposals through enforcement of explicit shape constraints inferred from the chosen Atlas ${ }_{a}$.

Though other approaches have been examined, the Point Distribution Model from Cootes \& Taylor ([1], [2]) seems to best suit our purpose. It requires an input training set of $n_{s}$ instances of studied object, where the same $n$ labeled landmarks have been placed to key anatomical loci characterizing the object's shape ( $n$ includes intermediate contour points). Principal Component Analysis (PCA) is then applied to resulting $n_{s}$ shape vectors of dimension $n_{p}=$ $2 \| 3 \times n$ to extract most significant linear variation modes of object's shape along the training set. They define an ellipsoidal $n_{p}-D$ Allowable Shape Domain (ASD) determining the space of variation - centered to mean shape - allowed to an unseen shape, thus providing a criterion for discriminating unlikely shapes.

Although this PDM model can represent shape of objects of any dimension, its applications (not only confined to Brain MRI) are far more frequent in 2D than in 3D considering that building an annotated training set in $3 \mathrm{D}$ raises new problems we attempted to overcome.

\section{TOWARDS AUTOMATIC 3D PDM BUILDING}

\subsection{Automatic building of a $3 D$ training set}

In Brain MRI domain, proper 2D PDMs can usually be achieved using a limited number of training shapes (e.g. $\simeq$ 20) demanding reasonable expert effort. But modeling of 3D shape variations generally requires a higher $n_{s}$, which is dramatic considering building one instance requires expert segmentation on about 50 volume slices.

\footnotetext{
${ }^{3}$ applying current method on $R e f_{b \neq a}$ and comparing to Atlas
} 
In our previous method, we noticed that the warp registration of $A_{t l a s_{a}}$, due to its global-consistent nature enabling local adaptations, managed to preserve the shape of our structures while still allowing them good fitting to target MRI. Due to residual errors, the resulting structures could not be considered as a final segmentation result, but close examination showed that these errors remained surprisingly satisfactory on most MRIs.

An encouraging fact is that after rigid realignment, these structures showed noticeable shape variations that should be learned by a PDM. Although exactness of these structures according to original MRI can be discussed, it should be compensated in PDM sense by the a priori unlimited number of MRIs $(\simeq 4000)$ available through Cyceron, though visual control is advised to reject a minority $(\simeq 20 \%)$ of poorly registered structures.

As a consequence, we will assume we can use these shape instances as a training set for our PDM model in a Bootstraping approach, considering that the segmentation results of our upcoming PDM-based method might refine the quality of the input set, until accordance to expert results converges to a satisfactory degree.

\subsection{Automatic landmarking using the MDL principle}

For similar reasons, if 2D landmarking can sometimes be manually practicable over limited sets, this generally does not scale to the 3D case through simple $z$-iteration, as we could experiment. Despite special cases with shapes of regular form, 3D shape variation should be considered globally, and often goes beyond simple observation or intuition. Some automatic methods were developed to detect noticeable points from 2D - and sometimes 3D - objects, but they tend to specialize to studied objects classes. Furthermore, none can state that these methods choose points intending to design a 'good' PDM.

Though, recent work from Rhodri Davies [2] formulated training set landmarking as a global optimization process. Each shape is mapped onto a corresponding sphere where a given number of landmarks is first evenly disposed. Then, their positions are blindly altered by reparameterization functions. Their evolution is regulated by an objective function evaluating the 'quality' of the PDM inferred by back-projection of displaced landmarks onto shapes. Thus, the whole process converges to the 'best possible' PDM for current training set.

The aforementioned objective function relies on the Minimum Description Length principle, which can be summed up as follows. Considering PCA analysis projects shape points - actually deviations from mean shape - to the space defined by computed orthogonal eigenvectors, we can assume the training set gets encoded by a centered multivariate Gaussian model. The idea, taken from the communication field, consists in attempting to pack together both model parameters and model-encoded values as a single message in the most compact form. Balancing model complexity and accuracy regarding to original data, DL is supposed to be minimal when the current training set interpretation - i.e. landmarking - is most both generalizable and compact. Results show better inferred PDMs than via manual landmarking, which often attempts to locate known points of anatomical significance, thus introducing a part of human subjectivity or inadaptation to current training set.

\section{RESULTS AND EXTENSIONS}

We successfully performed automatic landmarking on independent structures thanks to the program kindly provided by [3] that implements the method of [2] with some variations. Success of the whole procedure is suggested by distinct major variation modes that generate "correct" shape instances.

The exhaustive computation of the PDM model from a set of 27 brain MRIs takes approximatively 6 hours (annotation is just the final step): this remains fairly acceptable considering that this computation is only performed once for all.

The resulting PDM model will now be used to have the segmentation process respect the shape characteristics inferred from the training set: the use of ASMs or AAMs [1] is then considered with interest.

\section{ACKNOWLEDGMENTS}

We would like to thank Cyceron center for their support in providing data, and Allan Reinhold Kildeby and Rasmus Larsen from IMM who granted access to their 3D landmarking program and their advice.

\section{REFERENCES}

[1] T. Cootes and C. Taylor. Statistical models of appearance for medical image analysis and computer vision. In SPIE Medical Imaging, 2001.

[2] R. H. Davies, C. J. Twining, T. F. Cootes, J. C. Waterton, and C. J. Taylor. A minimum description length approach to statistical shape modeling. IEEE Transactions on Medical Imaging, 21(5):525-537, May 2002.

[3] A. R. Kildeby. Building optimal 3d shape models. Master's thesis, Informatics and Mathematical Modelling, Technical University of Denmark, DTU, 2002. Supervisor: Rasmus Larsen.

[4] J.-H. Xue, S. Ruan, B. Moretti, M. Revenu, and D. Bloyet. Knowledge-based segmentation an labeling of brain structures from mri images. Pattern Recognition Letters, 22:395-405, 2001. 